\title{
Solving the undirected feedback vertex set problem by local search
}

\author{
Shao-Meng Qin and Hai-Jun Zhou* \\ State Key Laboratory of Theoretical Physics, Institute of Theoretical Physics, \\ Chinese Academy of Sciences, Beijing 100190, China
}

(Dated: April 9, 2018)

\begin{abstract}
An undirected graph consists of a set of vertices and a set of undirected edges between vertices. Such a graph may contain an abundant number of cycles, then a feedback vertex set (FVS) is a set of vertices intersecting with each of these cycles. Constructing a FVS of cardinality approaching the global minimum value is a optimization problem in the nondeterministic polynomial-complete complexity class, therefore it might be extremely difficult for some large graph instances. In this paper we develop a simulated annealing local search algorithm for the undirected FVS problem. By defining an order for the vertices outside the FVS, we replace the global cycle constraints by a set of local vertex constraints on this order. Under these local constraints the cardinality of the focal FVS is then gradually reduced by the simulated annealing dynamical process. We test this heuristic algorithm on large instances of Erödos-Renyi random graph and regular random graph, and find that this algorithm is comparable in performance to the belief propagation-guided decimation algorithm.

PACS numbers: 89.20.Ff, 02.70.Uu, 02.10.Ox, 75.10.Nr
\end{abstract}

\section{INTRODUCTION}

An undirected graph is formed by a set of vertices and a set of undirected edges, with each edge connecting between two different vertices. A feedback vertex set (FVS) for such a graph is a set of vertices intersecting with every cycle of the graph. In other words, the subgraph induced by the vertices outside the FVS contains no cycle (it is a forest) [1, 2]. The feedback vertex set problem aims at constructing a FVS of small cardinality for a given undirected graph. It is a fundamental nondeterministic polynomial-complete (NP-complete) combinatorial optimization problem with global cycle constraints [1. 3. 4]. In terms of complete algorithms, whether a graph $G$ has a FVS of cardinality smaller than $n$ can be determined in time $O\left(3.592^{n}\right)$ [5]. And an FVS of cardinality at most two times the optimal value can be easily constructed by an efficient polynomial algorithm [8]. An optimal FVS is a feedback vertex set whose cardinality is the global minimum value among all the feedback vertex sets of the graph. For a given graph, an optimal FVS can be constructed in an exact way in time $O\left(1.7548^{N}\right)$ [9, 10, where $N$ denotes the total number of vertices in the graph. Applied mathematicians have obtained rigorous lower and upper bounds for the optimal FVS problem [1] and have proved its tractability for graphs with specific structures (see for example, [12, 13] and references cited therein).

Due to the NP-complete nature of the FVS problem, in general it is not feasible to construct optimal feedback vertex sets for large cycle-rich graphs. An important question is then to design efficent heurstic algorithms that are able to obtain near-optimal FVS solutions for given graph instances. Such a task is quite nontrivial. A

\footnotetext{
${ }^{*}$ Corresponding author. Email address: zhouhj@itp.ac.cn.
}

major technical difficulty is that cycles are global objects of a graph and therefore the existence of cycles can not be judged by checking only the neighborhood of a vertext. (Similar difficulties exist in other combinatorial optimization problems with global constraints, such as the Steiner tree problem [14] and the optimal routing problem 15, 16.) In Ref. [17, one of the authors succeeded in converting the FVS problem to a spin glass problem with local interactions. The FVS problem was then studied from the spin glass perspective, and a message-passing algorithm, belief propagation-guided decimaton (BPD), was impletmented to solve the FVS problem heuristically for single graph instances. This BPD algorithm is quite efficient in terms of computing time and computer memory (since there is no need of cycle checking), and it can obtain FVS solutions that are very close to the optimal ones when applied on large random graph instances and regular lattices [17.

For the undirected FVS problem it is not yet known whether simple local search algorithms can achieve equally excellent results as the BPD algorithm. Motivated by this question, we complement the messagepassing approach in this paper by implementing and testing a simulated annealing local searching (SALS) protocol [18] for the undirected FVS problem. A similar algorithmic study has already been undertaken in [19] for directed graphs. Here we modify the microscopic search rules of [19 to make it applicable to undirected graphs. In the SALS algorithm, an order is defined for the vertices outside the focal FVS, and this order is constrained by a set of local vertex constraints. Our simulation results suggest that this local search algorithm is comparable in performance to the BPD algorithm at least for random graph instances.

The feedback vertex set problem has wide practical applications in the field of computer science (such as integrated circuit design and database management). Although not yet seriously explored, the FVS problem may 


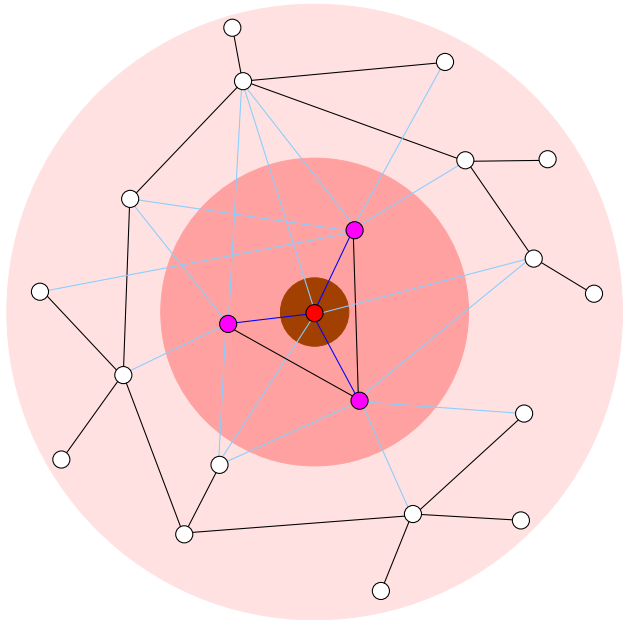

FIG. 1: (color online). The four filled points form an optimal feedback vertex set for this graph $G$. The subgraph $g$ induced by these four vertices and the cycle-free subgraph induced by all the remaining vertices (shown as open points) are connected through many edges (shown in light blue). Since the subgraph $g$ still contains cycles within itself, we decompose it into a tree subgraph of three vertices (filled magenta points) and a subgraph formed by a FVS of one vertex (the central red point). By this way, the vertices in the orginal graph $G$ are arranged into three different layers. The vertices of each layer form a cycle-free subgraph (a tree or a forest), while different layers are connected by edges. An important property of such an organization is that each cycle must involves vertices from at least two layers.

have many potential applications in complex systems research as well. For example, if a vertex is contained in a large fraction of the near-optimal feedback vertex sets, we may expect this vertex to play a very significant role for the dynamical processes on the graph. Therefore the probability of belonging to a near-optimal FVS can serve as a centrality index of dynamical significance for each vertex of a graph. Such a probablity can be computed by sampling many independent near-optimal FVS solutions or be computed directly using the belief propagation iterative equations [17.

The construction of a near-optimal FVS also facilitates the study of a complex dynamical system as a simpler response problem of a cycle-free subsystem (which is intrinsically simple) under the influence of the vertices in the FVS vertices. If the subgraph induced by the vertices in the FVS itself contains many cycles, such a decomposition can be applied on this subgraph again. Through this iterated process, an initial cycle-rich complex graph is then organized into a hierarchy of forest (cycle-free) subgraphs and the edges between these forests. A simple illustration of this hierarchical organization is shown in Fig. 1. We believe such a hierarchical representation of a complex graph will be very helpful in future dynamical applications.

The next section describes the SALS algorithm in de- tail, and in Sec. 3 we test the performance of this local search algorithm on random graph instances and compare the results with the results obtained by the BPD algorithm and those obtained by the replica-symmetric mean field theory. We conclude this work in Sec. 4. The two appendices are the proofs of the theorems of Sec. 2 .

\section{THE LOCAL SEARCH ALGORITHM}

For a graph $G$ of $N$ vertices, let us consider an ordered list $L$ formed by $n \leq N$ vertices of this graph

$$
L \equiv\left(v_{1}, v_{2}, \ldots, v_{n}\right)
$$

Following [19, we assign to the first vertex $v_{1}$ of this list an integer rank $r=1$, to the second vertex an integer rank $r=2, \ldots$, and to the last vertex $v_{n}$ an integer rank $r=n$. Therefore each vertex $i \in L$ has an integer rank $r_{i}$ which marks the position of this vertex in the list $L$. For the purpose of the FVS problem, we introduce for each vertex $i \in L$ a ranking condition as

$$
\sum_{j: j \in L,(i, j) \in G} \Theta\left(r_{i}-r_{j}\right) \leq 1,
$$

where $(i, j)$ denotes an edge of the graph $G$ between vertex $i$ and vertex $j$, and $\Theta(x)=1$ if $x>0$ and $\Theta(x)=0$ if $x \leq 0$. The ranking condition (2) is satisfied by vertex $i$ if and only if among all the nearest neighboring vertices of vertex $i$ that are also contained in the list $L$, at most one of them has a lower rank than that of $i$. A list $L$ is referred to as a legal list if all its vertices satisfy the ranking condition (2). The link between legal lists and feedback vertex sets is setup by the following two theorems:

Theorem 1 If $L$ is a legal list, then the subgraph of the graph $G$ induced by all the vertices of this list is cycle-free. Therefore the set $\Gamma$ formed by all the remaining vertices of $G$ not included in $L$ is a FVS.

Theorem 2 If $\Gamma$ is a FVS for a graph $G$, then it is possible to form a legal list $L$ using all the vertices not contained in $\Gamma$.

These two theoretms are easy to prove, see the appendices for technical details. They suggest that there is a one-to-many correspondance between a FVS $\Gamma$ and legal lists $L$. Therefore the problem of constructing an optimal FVS is converted to a problem of constructing a legal list $L$ of maximal cardinality. Notice that judging whether a list $L$ is legal or not is algorithmically very easy as Eq. (2) involves only the neighborhood a focal vertex $i$ but not the connection pattern of the whole graph $G$. A similar conversion from global constraints to local constraints has also been used in the Steiner tree problem, which aims at constructing a tree of minimal total edge length connecting a set of specified vertices [14, 20]. 
Just for simplicity of later discussions, let us define the energy of a legal list $L$ as the total number of vertices not contained in it, namely

$$
E(L) \equiv N-\sum_{i \in L} 1 .
$$

In otherwords, $E(L)$ is just the cardinality of the complementary FVS $\Gamma$ of the list $L$. Following Ref. 19] we implement a simulated annealing local search algorithm as follows:

0 . Input the graph $G$. Initialize the legal set $L$ as containing only a single randomly chosen vertex of $G$, and the complementary feedback vertex set $\Gamma$ then contains all the other vertices of $G$. Set the temperature $T$ to an initial value $T_{0}$.

1. Choose a vertex (say $i$ ) uniformly at random from the feedback vertex set $\Gamma$.

(a) If the list $L$ contains no nearest neighbor of the vertex $i$, delete $i$ from $\Gamma$ and insert it to the head of $L$. Then vertex $i$ has rank $r_{i}=1$ in the updated list $L$ and the energy of $L$ decreases by 1 .

(b) If $L$ contains exactly one nearest neighbor (say $j$ with rank $r_{j}$ ) of the vertex $i$, delete $i$ from $\Gamma$ and insert it to $L$ at the position just after vertex $j$. Then vertex $i$ has rank $r_{i}=r_{j}+1$ in the updated list $L$ and the energy of $L$ decreases by 1 .

(c) If $L$ contains two or more nearest neighbors (say $j, k, \ldots$ with vertex $j$ having the lowest rank $r_{j}$ among these vertices) of the vertex $i$, we make a proposal of moving $i$ from $\Gamma$ to the ordered list $L$ at the position just after vertex $j$ and deleting all those nearest neighbors of $i$ from $L$ if the insertion of $i$ causes the violation of the ranking condition 2 for these vertices. Suppose $n_{d} \geq 0$ vertices have to be deleted from $L$ (and be added to $\Gamma$ ) as a result of inserting vertex $i$ to $L$, then the energy increase of $L$ is $\Delta E=-1+n_{d}$. If $\Delta E \leq 0$ we accept the proposed action with probability 1 , otherwise we accept it with probability $\exp (-\Delta E / T)$. If this proposal is accepted, then vertex $i$ has rank $r_{i}=r_{j}+1$ in the updated list $L$.

2. Repeat step 1 until the list $L$ has been successfully updated for $N_{t}$ times. During this process, record the best FVS so far reached and the corresponding lowest energy, $E_{\min }$.

3. Decrease the temperature to $T \leftarrow T \times \alpha$, where $\alpha<1$ is a fixed constant, and then repeat steps 1-2. If the energy value $E_{\text {min }}$ does not change in $N_{\text {fail }}$ contiguous temperature stages, then we stop the local search process and output the reached best FVS.

In our computer experiments we use $T_{0}=0.6, N_{t}=50$ and $N_{\text {fail }}=50$ which are identical to the values used in
Ref. [19]. The temperature ratio $\alpha$ is set to several different values $\alpha=0.99, \alpha=0.98$ and $\alpha=0.9$. As shown in Fig. 2, the slower the rate of temperature decrease, the lower is the energy of the constructed best FVS solutions.

\section{NUMERICAL RESULTS}

The BPD algorithm was applied to Erdös-Renyi (ER) random graphs and regular (RR) random graphs in [17] to test its performance. As we want to compare the performance of the present SALS algorithm with the BPD algorithm, we apply the SALS algorithm on the same ER and $\mathrm{RR}$ random graphs used in [17.

An ER random graph of $N$ vertices and $M=(c / 2) N$ edges was generated in [17] by first selecting $M$ different vertex pairs uniformly at random from the whole set of $N(N-1) / 2$ candidate vertex pairs and then connecting the two vertices of each selected vertex pair by an edge. A vertex in such a graph has on average $c$ nearest neighbors (i.e., the mean vertex degree of the graph is $c$ ). When $N \gg 1$ the vertex degree distribution of the graph converges to a Poisson distribution of mean value $c$ [21]. A RR graph differs from an ER graph with the additional constraint that each vertex has exactly the same number $c$ of nearest neighbors (here $c$ must be an integer). It was generated by first attaching to each vertex a number of $c$ half-edges and then randomly connecting two half-edges into a full edge, but prohibiting self-loops or multiple edges between the same pair of vertices (see Ref. 22, 23. for more details of the graph generation process).

The vertex number of all these random graph instances is equal to the same value $N=10^{5}$. There are 96 independently generated $\mathrm{ER}$ or RR random graph instances at each fixed (mean) degree value $c$. We run the SALS process once on each of these 96 instances and then compare the mean value of the obtained final $E_{\min }$ values with the mean value of the final energies obtained in [17] by running the BPD process once on each of these 96 graph instances.

The evolution of the minimal energy $E_{\min }$ as a function of the temperature $T$ is shown in Fig. 2 for ER (a) and $\mathrm{RR}(\mathrm{b})$ random graphs of $c=10$. When $T>0.4$ the evolution curves for the three different cooling parameters $\alpha=0.9,0.9801$ and 0.99 coincide with each other. This indicates that at $T>0.4$ the typical relaxation time of energy is shorter than the time scale of temperature decreasing. At $T \approx 0.4$ the curve of $\alpha=0.9$ starts to separate from the other two curves, and the final value of $E_{\min }$ is considerably higher than those of the other two evolutionary trajectories. This indicates that at cooling parameter $\alpha=0.9$ the simulated annealing process is eventually trapped in a local region of the configuration space whose energy minimal value is extensively higher than that of the optimal solutions. The two evolutionary curves of $\alpha=0.9801$ and $\alpha=0.99$ again separate from each other at a lower temperature of $T \approx 0.35$, and the final value of $E_{\min }$ reached at cooling parameter $\alpha=0.99$ 


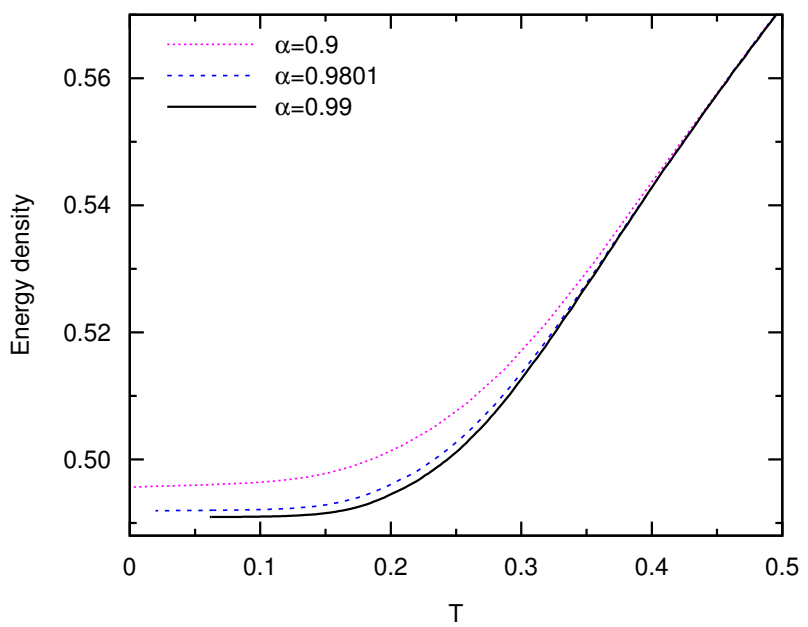

(a)

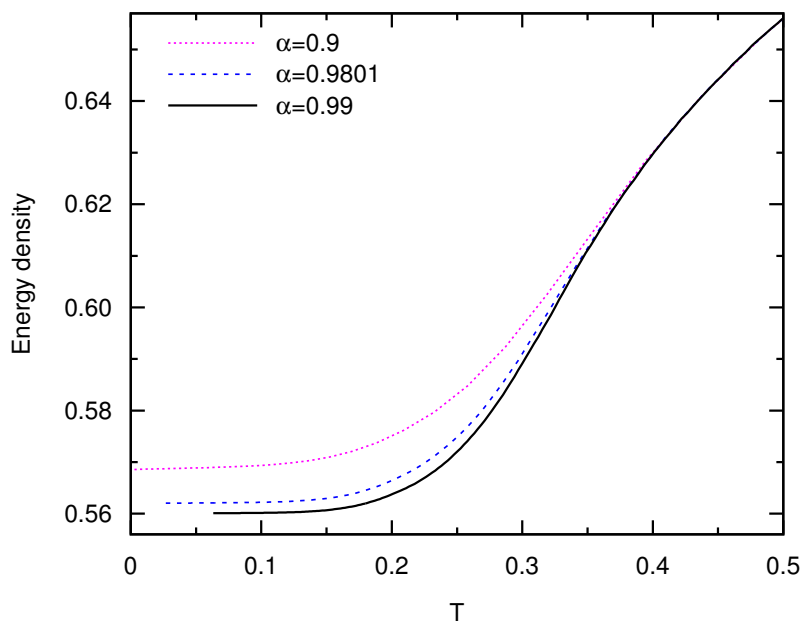

(b)

FIG. 2: Evolution of the minimal energy density. The minimal energy density is equal to $E_{\min } / N$, where $E_{\text {min }}$ is the lowest energy so far reached during the annealing process from the initial temperature $T_{0}=0.6$ to the current value $T$. Each curve is the averaged result of a single run of the SALS algorithm on 96 random graph instances of vertex number $N=10^{5}$. (a) ER random graphs of mean vertex degree $c=10$. (b) RR random graphs of vertex degree $c=10$.

is slightly lower than the final value of $E_{\min }$ reached at $\alpha=0.9801$. If the value of $\alpha$ is set to be more closer to 1 the final value of $E_{\text {min }}$ will decrease slightly further (at the expense of much longer simulation times). The observation that lower final energy values can be reached by lowering the cooling rate strongly indicates the system has a very complicated low-energy landscape with many local minima. This is consistent with the prediction that the undirected FVS problem is in a spin glass phase at low enough energy values [17.

Figure 3 compares the performance of the SALS algorithm (the cooling parameter fixed to $\alpha=0.99$ ) with that of the BPD algorithm on ER (a) and RR (b) random

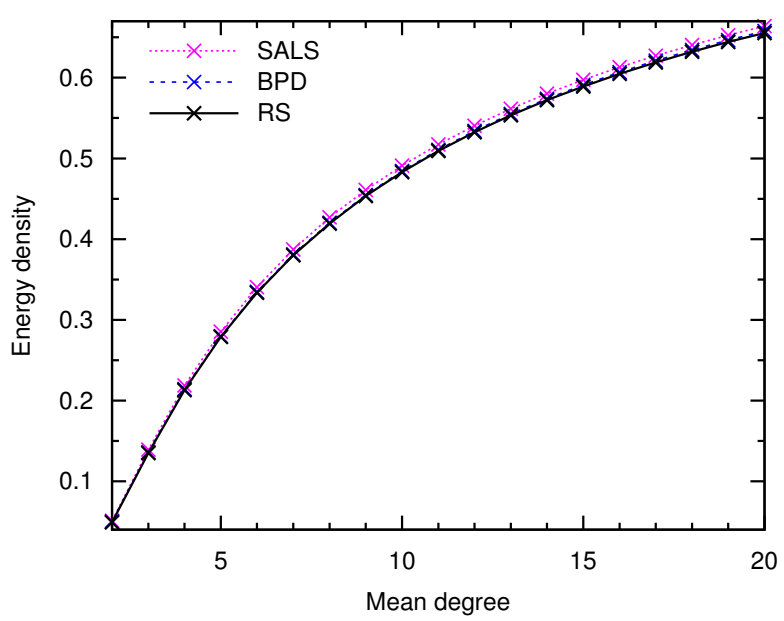

(a)

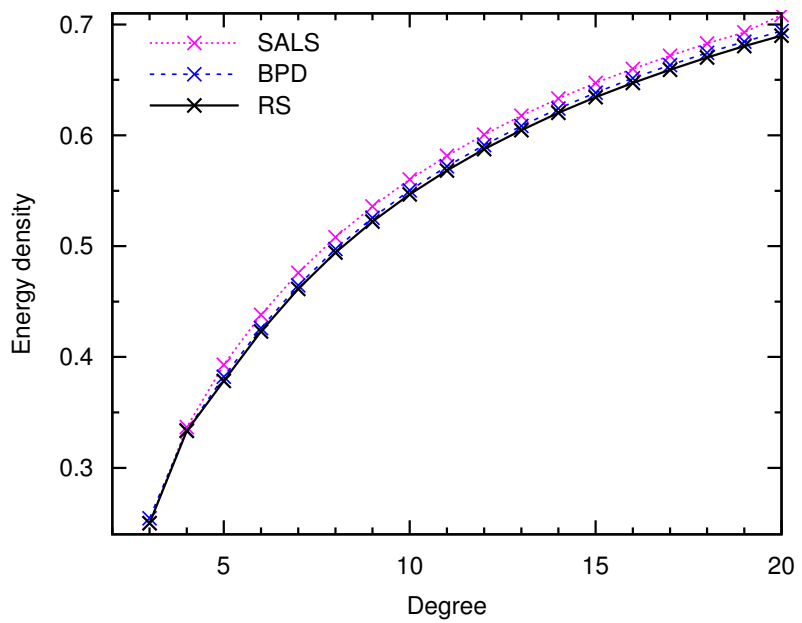

(b)

FIG. 3: The energy density (i.e., relative size) of feedback vertex sets for ER (a) and RR (b) random graphs. The SALS and BPD algorithms are applied to 96 random graph instances of $N=10^{5}$ at each value of (mean) vertex degree $c$. The averaged results over these 96 instances are shown here (the standard deviations are smaller than the size of the symbols and are not shown for clarity). As a comparison we also show the results obtained by the replica-symmetric (RS) mean field theory at $N=\infty$.

graphs. We see that BPD slightly outperforms SALS for both ensembles of random graphs. This is not surprising, since the BPD algorithm takes into account the global structure of the graph through message-passing, while the SALS algorithm considers only the local graph structure. It is interesting to see that the results of the two algorithms are actually very close to each other, especially for ER random graphs. As also shown in Fig. 3 . at each value of (mean) degree $c$, the result obtained by the SALS algorithm is very close to the predicted value of the global minimal energy by the replica-symmetric mean field theory [17. 


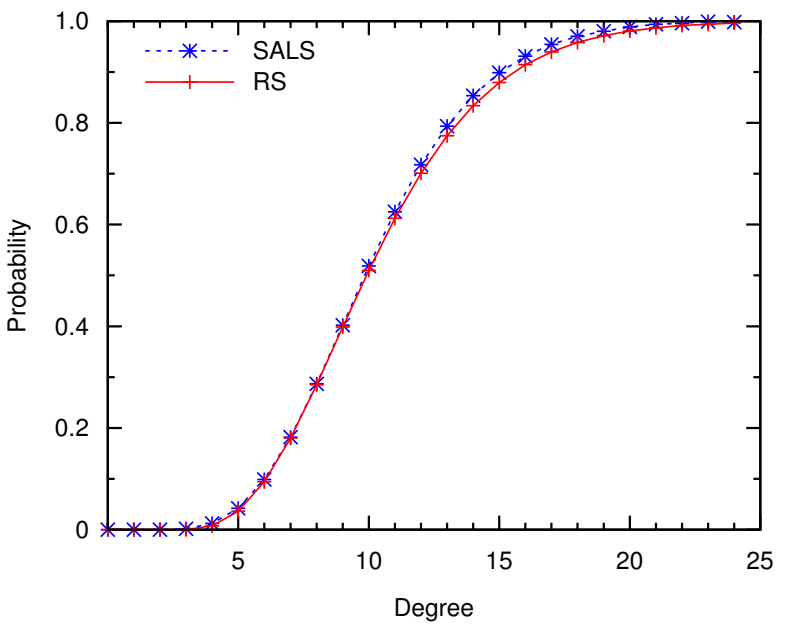

FIG. 4: In an ER random graph of mean degree $c=10$, a vertex's probability of being in a feedback vertex set increases with its vertex degree $k$. Each star point is the mean result obtained by averaging over the 96 final FVS solutions obtained by the SALS algorithm on the 96 random graph instances of $N=10^{5}$ vertices. Each plus point denotes the result obtained by the RS mean field theory at $N=\infty$ 17.

Therefore message-passing algorithms are not the only candidate choices to construct near-optimal feedback vertex sets for undirected random graphs. An advantage of the SALS algorithm is that its implementation is very straightforward. It may be the method of choice for many practical applications.

We also notice from Fig. 3 that the SALS algorithm performs poorer in regular random graphs than in ER random graphs. This is probably due to the following fact: Each vertex in a regular random graph has the same degree so there is local guide as to whether a vertex should be included into the feedback vertex set or not. On the other hand the degree heterogeneity of an ER graph will give some local guide to the SALS process to arrive at a near-optimal FVS. Based on the final 96 FVS solutions obtained by the SALS algorithm on the 96 ER random graphs with mean vertex degree $c=10$, we compute the mean probability $f_{k}$ that a vertex of degree $k$ is contained in the FVS. The results shown in Fig. 4 demonstrate that $f_{k}$ is close to 0 for $k \leq 4$ and close to 1 for $k \geq 20$, and it is an rapidly increasing function of $k$ in the range of $5 \leq k \leq 15$. The empirically obtained mean value of $f_{k}$ is found to be very close to the value of $f_{k}$ computed using the replica-symmetric mean field theory. Notice that for $10 \leq k \leq 20$ the empirical values of $f_{k}$ are slightly larger than those predicted by the RS mean field. Such small differences cause the energies of the construsted FVS solutions to be higher than the corresponding optimal values.

The graphs for real-world complex systems usually are highly heterogenous in terms of vertex degree distributions 21. Therefore it is very likely that the SALS algorithm to have very good performance for such graphs.

\section{CONCLUSION}

In this paper, we implemented and tested a local search algorithm for the undirected feedback vertex set problem. Similar to the local search algorithm for the directed FVS problem [19, our algorithm uses the technique of simulated annealing to explore the highly complex landscape of low-energy congiurations. Our simulation results demonstrated that this algorithm is very efficient for large random graph instances. The relative sizes of the constructed FVS solutions by the local search algorithm are very close to the predicted values of the replicasymmetric mean field theory, and they are also very similar to the results obtained by the BPD message-passing algorithm.

It should be emphasized that the microscopic dynamical rules of our local search algorithm do not obey the detailed balance condition. These dynamical rules therefore need to be appropriately modified if one is interested in the equilibrial FVS solutions at a given fixed temperature $T$. We are currently using a modified set of microscopic dynamical rules to study the spin glass phase transition of the model proposed in [17. Such an equilibrium study will offer more physical insights on the cooling-rate dependent behaviors of Fig. 2 .

\section{Acknowledgement}

The numerical simulations were performed at the HPC computer cluster of the authors' institute. This work was partially supported by the National Basic Research Program of China (No. 2013CB932804), the Knowledge Innovation Program of Chinese Academy of Sciences (No. KJCX2-EW-J02), and the National Science Foundation of China (grant Nos. 11121403, 11225526). HJZ conceived research; SMQ performed research; HJZ wrote the paper.

\section{Appendix A: Proof of Theorem 1}

Consider a generic legal list $L$ formed by some vertices of the graph $G$, see Eq. (1). Let us consider the subgraph $F$ induced by all the vertices of list $L$ and all the edges between these vertices. Since $L$ is a legal list, every vertex $i \in L$ must satisfy the rank condition (2). Consequently, every vertex in the subgraph $F$ has at most one nearest neighbor with rank lower than that of itself.

This subgraph $F$ must be cycle-free. We prove this statement by contradiction. Assume there is a cycle in the subgraph $F$ involving $k$ vertices:

$$
\left(i_{1}, i_{2}\right),\left(i_{2}, i_{3}\right), \ldots,\left(i_{k-1}, i_{k}\right),\left(i_{k}, i_{1}\right) .
$$

If the rank of vertex $i_{1}$ is lower than that of $i_{2}$, then due to the fact of vertex $i_{2}$ having at most one nearest neighbor with lower rank than itself, the rank of $i_{3}$ must 
be higher than that of $i_{2}$. Continuing this analysis along the cycle, we obtain that the rank of vertex $i_{k}$ must be higher than that of $i_{k-1}$ but lower than that of $i_{1}$. But this is impossible since the rank of $i_{k-1}$ must be higher than that of $i_{1}$. Similarly, if the rank of vertex $i_{1}$ is higher than that of $i_{2}$, we will arrive at the contradicting results that the rank of $i_{2}$ is higher than that of $i_{3}$ while the rank of $i_{3}$ is higher than that of $i_{1}$. Because of these contradictions, the assumption of $F$ containing a cycle must be false.

Therefore $F$ must be a tree or a forest. Then the set $\Gamma$ formed by the vertices not contained in $L$ must be a feedback vertex set.

\section{Appendix B: Proof of Theorem 2}

Suppose the set $\Gamma$ is a FVS of a graph $G$. Then the subgraph $F$ induced by all the vertices not included in $\Gamma$ must be cycle-free.

If $F$ has only one connected component (i.e., being a tree), we can pick a vertex (say $i$ ) of $F$ uniformly at random and specify this vertex as the root of the tree subgraph $F$. We can then construct an ordered list $L$ using all the vertices of $F$ in such a way: first the root vertex $i$, then all the vertices of unit path length to $i$ (in random order), followed by all the vertices of path length two to $i$ (again in random order), ..., followed by the remaining vertices of the longest path length to $i$ (in random order). Obviously $L$ is a legal list with the ranking condition (2) satisfied for all its vertices.

If $F$ is a forest with two or more tree components, we can perform the above-mentioned process for each of its tree components and then contatenate the constructed ordered lists in a random order to form a whole ordered list $L$. This list $L$ must also be a legal list.
[1] Garey M, Johnson DS (1979) Computers and Intractability: A Guide to the Theory of NP-Completeness. (Freeman, San Francisco).

[2] Festa P, Pardalos PM, Resende MGC (1999) in Handbook of combinatorial optimization, eds. Du DZ, Pardalos PM. (Springer, Berlin, Germany), pp. 209-258.

[3] Karp RM (1972) Reducibility among combinatorial problems eds. Miller E, Thatcher JW, Bohlinger JD. (Plenum Press, New York), pp. 85-103.

[4] Cook SA (1971) The complexity of theorem-proving procedures eds. Lewis PM et al. (ACM, New York), pp. $151-158$

[5] Cao Y, Chen J, Liu Y (2010) On feedback vertex set new measure and new structures. Lect. Notes Comput. Sci. 6139:93-104.

[6] Kociumaka T, Pilipczuk M (2013) Faster deterministic feedback vertex set (arXiv:1306.3566v1).

[7] Guo J, Gramm J, Hüffner F, Niedermeier R, Wernicke S (2006) Compression-based fixed-parameter algorithms for feedback vertex set and edge bipartization. J. Comput. System Sci. 72:1386-1396.

[8] Bafna V, Berman P, Fujito T (1999) A 2-approximation algorithm for the undirected feedback vertex set problem. SIAM J. Discrete Math. 12:289-297.

[9] Razgon I (2006) Exact computation of maximum induced forest. Lect. Notes Comput. Sci. 4059:160-171.

[10] Fomin FV, Gaspers S, Pyatkin AV (2006) Finding a minimum feedback vertex set in time $o\left(1.7548^{n}\right)$. Lect. Notes Comput. Sci. 4169:184-191.

[11] Bau S, Wormald NC, Zhou S (2002) Decycling numbers of random regular graphs. Random Struct. Alg. 21:397413.

[12] Wang FH, Wang YL, Chang JM (2004) Feedback ver- tex sets in star graphs. Information Processing Letters 89:203-208.

[13] Jiang W, Liu T, Xu K (2011) Tractable feedback vertex sets in restricted bipartite graphs. Lect. Notes Comput. Sci. 6831:424-434.

[14] Bayati M et al. (2008) Statistical mechanics of steiner trees. Phys. Rev. Lett. 101:037208.

[15] Yeung CH, Saad D, Wong KYM (2013) From the physics of interacting polymers to optimizing routes on the london underground. Proc. Natl. Acad. Sci. USA 110:1371713722 .

[16] Yeung CH, Saad D (2013) Networking-a statistical physics perspective. J. Phys. A: Math. Theor. 46:103001.

[17] Zhou HJ (2013) Spin glass approach to the feedback vertex set problem. Eur. Phys. J. B 86:455.

[18] Kirkpatrick S, Gelatt Jr. CD, Vecchi MP (1983) Optimization by simulated annealing. Science 220:671-680.

[19] Galinier P, Lemamou E, Bouzidi MW (2013) Applying local search to the feedback vertex set problem. J. Heuristics 19:797-818.

[20] Biazzo I, Braunstein A, Zecchina R (2012) Performance of a cavity-method-based algorithm for the prizecollecting steiner tree problem on graphs. Phys. Rev. E 86:026706.

[21] He DR, Liu ZH, Wang BH (2009) Complex Systems and Complex Networks. (Higher Education Press, Beijing).

[22] Zhou HJ, Lipowsky R (2007) Activity patterns on random scale-free networks: global dynamics arising from local majority rules. J. Stat. Mech.: Theor. Exp. p. P01009.

[23] Zhou HJ, Lipowsky R (2005) Dynamic pattern evolution on scale-free networks. Proc. Natl. Acad. Sci. USA 102:10052-10057. 